\title{
All-order symmetric subtraction of divergences for massive Yang-Mills theory based on non linearly realized gauge group
}

\author{
Andrea Quadri* \\ Università di Milano \& INFN, Sez. di Milano \\ E-mail: andrea.quadri@mi.infn.it
}

\section{Daniele Bettinelli}

Università di Milano \& INFN, Sez. di Milano

E-mail: daniele.bettinelli@mi.infn.it

\section{Ruggero Ferrari}

Università di Milano \& INFN, Sez. di Milano

E-mail: ruggero.ferrari@mi.infn.it

We review a recently proposed all-orders symmetric subtraction procedure for massive YangMills theory based on the nonlinearly realized gauge group. Physical unitarity is fulfilled order by order in the loop expansion. The theory is defined in terms of a finite number of physical parameters entering in the tree-level vertex functional. An outlook on future developments is also given.

8th International Symposium on Radiative Corrections

October 1-5, 2007

Florence, Italy

\footnotetext{
* Speaker.
} 
The mass generation by the spontaneous symmetry breaking implemented via the Higgs mechanism is the last yet untested pillar of the Standard Model. A sharp prediction of this theoretical scheme is the existence of (at least one) additional physical scalar particle.

In this connection it is a remarkable fact that an alternative spontaneous symmetry breaking procedure has been known since a long time which avoids (perturbatively) the introduction of any additional physical particle in the spectrum. This goes under the name of the Stiickelberg mechanism [1],[2] and is based on a nonlinearly realized representation of the non-Abelian gauge group. However, a consistent formulation of this mechanism was known only at the classical level, and the issue of how to define the nonlinearly realized massive Yang-Mills theory at the quantum level has remained up to now an unsettled question. The reason is that the nonlinearly realized theory, unlike its linear counterpart, is not power-counting renormalizable and therefore the standard subtraction procedures, developed in the context of gauge theories during the last quarter century, cannot be applied.

Recently a consistent theoretical framework for the all-orders subtraction of nonlinearly realized massive SU(2) Yang-Mills theory has been achieved in [3]. In this formulation one introduces the SU(2) element

$$
\begin{aligned}
& \Omega=\frac{1}{v}\left(\phi_{0}+i \tau_{a} \phi_{a}\right), \quad \Omega^{\dagger} \Omega=1, \quad \operatorname{det} \Omega=1, \\
& \phi_{0}^{2}+\phi_{a}^{2}=v^{2}
\end{aligned}
$$

where $v$ is the spontaneous symmetry breaking parameter. $\phi_{a}$ are the pseudo-Goldstone fields.

The gauge group flat connection is defined by

$$
F_{\mu}=i \Omega \partial_{\mu} \Omega^{\dagger}=F_{a \mu} \frac{\tau_{a}}{2},
$$

where $\tau_{a}$ are the Pauli matrices. The $S U(2)_{L}$ gauge transformation on $\Omega$ and the gauge connection $A_{\mu}=A_{a \mu} \frac{\tau_{a}}{2}$ is

$$
\begin{aligned}
& \Omega^{\prime}=U_{L} \Omega, \\
& F_{\mu}^{\prime}=U_{L} F_{\mu} U_{L}^{\dagger}+i U_{L} \partial_{\mu} U_{L}^{\dagger}, \\
& A_{\mu}^{\prime}=U_{L} A_{\mu} U_{L}^{\dagger}+i U_{L} \partial_{\mu} U_{L}^{\dagger} .
\end{aligned}
$$

The gauge-invariant Stückelberg mass term can be parameterized in $D$ dimensions as (we follow the conventions of [3])

$$
\frac{\Lambda^{D-4}}{g^{2}} \int d^{D} x \frac{M^{2}}{2}\left(A_{a \mu}-F_{a \mu}\right)^{2} .
$$

$\Lambda$ is the scale of the radiative corrections, $g$ is the gauge coupling constant and $M$ is the mass of the vector mesons.

The resulting model is not renormalizable by power-counting, due to the couplings arising in eq. (4) from the nonlinear constraint $\phi_{0}$ entering in $F_{a \mu}$. In fact severe UV divergences occur in the sector spanned by the pseudo-Goldstone fields, since already at one loop level there is an infinite number of divergent amplitudes with external pseudo-Goldstone legs. However it turns out that such a wild behaviour can be tamed by imposing a local functional equation [5] which encodes 
the invariance of the $\mathrm{SU}(2)$ Haar measure under local left multiplication. This equation was first studied for the SU(2) nonlinear sigma model in [5]-[9]. It gives rise to a bilinear local functional identity for the vertex functional $\Gamma$ yielding a powerful hierarchy among Green functions. The Green functions involving at least one pseudo-Goldstone field (descendant amplitudes) are fixed by the local functional equation once the amplitudes with no external pseudo-Goldstone legs (ancestor amplitudes) are known.

At each order in the loop expansion only a finite number of ancestor amplitudes are divergent. This result holds as a consequence of the weak power-counting theorem [3], [6]. The pole part of any amplitude can be reconstructed once one knows the pole part of the ancestor amplitudes either by directly solving the local functional equation or by making use of the cohomological methods discussed in [6] for the one loop approximation and in [9] at arbitrary order in the loop expansion.

These methods have been used in [4] in order to provide a compact representation of the oneloop counterterms of the nonlinearly realized massive Yang-Mills theory in terms of a suitable linear combination of invariants compatible with all the symmetries of the model.

In addition to the local functional equation a number of other identities exist in the case of the nonlinearly realized massive Yang-Mills theory.

The Slavnov-Taylor (ST) identity must be imposed in order to fulfill the requirement of physical unitarity [3]. The proof of the latter in the perturbative expansion has been given both in an operatorial approach based on the existence of a conserved BRST charge and in a diagrammatic approach based on the cutting rules [1]. It is worth noticing that the ST identity does not give rise to the hierarchy among Green functions required in order to control the divergences in the nonlinear theory. This important point is discussed at length in [3]. The local functional equation cannot be discarded in the program of subtracting the divergences of the nonlinearly realized massive YangMills. On the other hand, it is not a substitute for the ST identity as far as physical unitarity is addressed. Both of them are essential tools in achieving a consistent formulation of the theory at the quantum level.

The Landau gauge-fixing has been used in [3],[4]. The ghost equation and the Landau gauge equation allow to control the dependence on the gauge-fixing also in the nonlinearly realized case. The extension to an arbitrary 't Hooft gauge requires some care in the nonlinear theory. The appropriate procedure is outlined in [10]. In the latter reference it is also shown that the gaugeindependent vector bosons self mass can be computed in the nonlinearly realized theory as the zero of the two-point vector bosons 1-PI function (unlike in the linear model where the inclusion of the tadpoles is required in order to fulfill the gauge independence). The explicit evaluation of the self mass for the nonlinear theory has been given in [4].

The subtraction procedure is based on the subtraction of the poles in $D-4$ of the amplitudes, in dimensional regularization, after a suitable normalization has been performed. The latter is essential in order to guarantee the fulfillment of the symmetries of the model.

The question of the number of physical parameters within the present approach has been addressed in [8]. The problem can be stated as follows. The removal of the divergences is controlled at each loop order by a set of invariants (with divergent coefficients in the limit $D \rightarrow 4$ ) compatible with the symmetries of the theory. These invariants are finite in number at each order in the loop expansion. The issue arises of whether a finite number of new independent physical parameters can be introduced order by order in the loop expansion in correspondence to the coefficients of such 
invariants. It turns out that this is not possible in the present approach, since these invariants cannot be inserted back into the tree-level vertex functional without violating either the symmetries or the weak power-counting theorem.

We wish to make some more comments on the violation of the weak power-counting. The presence of the flat connection allows to trivialize the $S U(2)_{L}$ left symmetry, since the following bleached combination

$$
a_{\mu}=\Omega^{\dagger} A_{\mu} \Omega-i \Omega^{\dagger} \partial_{\mu} \Omega=a_{a \mu} \frac{\tau_{a}}{2}
$$

is invariant under the gauge transformations in eq.(3). Therefore any polynomial in $a_{a \mu}$ and its derivatives is allowed by the nonlinearly realized gauge symmetry. However, the imposition of the validity of the weak power-counting strongly constraints the dynamics of the gauge fields. In fact the most general classical action compatible with the nonlinearly realized $S U(2)_{L}$ gauge symmetry and the weak power-counting theorem is [3]

$$
S=\frac{\Lambda^{(D-4)}}{g^{2}} \int d^{D} x\left(-\frac{1}{4} G_{a \mu v}[A] G_{a}^{\mu v}[A]+\frac{1}{2} M_{a b} a_{a \mu} a_{b}^{\mu}\right)
$$

where $G_{a \mu v}[A]$ is the field strength of $A_{a \mu}$ and $M_{a b}$ is an arbitrary symmetric mass matrix. Therefore the standard Yang-Mills term is recovered by the requirement of the validity of the weak powercounting. By imposing the global $S U(2)_{R}$ symmetry, the mass term in eq.(6) reduces to the diagonal Stückelberg term in eq.(4).

On the other hand, if the interaction terms of the gauge bosons are modified w.r.t. the standard Yang-Mills term by adding monomials in $a_{a \mu}$ and its derivatives, the weak power-counting is maximally violated (an infinite number of one-loop divergent ancestor amplitudes involving the gauge field $A_{a \mu}$ arise [3]) and the theory can never be made finite already at one loop.

The theory has two physical parameters: the gauge boson mass $M$ and the gauge coupling constant $g$. A change in $\Lambda$ cannot be compensated by a shift in $M$ and $g . \Lambda$ should be fitted against the comparison with the experimental data and plays the rôle of a further parameter setting the scale of the physical observables. $v$ is not a physical parameter [3].

The theoretical framework we have outlined is being developed by successive steps. It provides a consistent and theoretically robust set of tools for dealing with nonlinearly realized gauge theories at the quantum level. There are many important questions that could be addressed in the future. Among them we wish to mention the running of the coupling constants and the associated problem of the resummation, as well as the compatibility of the perturbation expansion with the Froissart bounds. With reference to the latter issue, the fulfillment of physical unitarity order by order in the loop expansion might imply an improvement in the range of energies where perturbation theory is reliable w.r.t. naive estimates based on dimensional arguments.

A somehow related but independent issue is the application of the present approach to the electroweak group $\mathrm{SU}(2) \mathrm{xU}(1)$. The subtraction procedure we have described would provide one consistent way to evaluate the radiative corrections driven by the nonlinearly realized gauge group, and eventually compare them with the results obtained in the linear model and with the experiments. This might become in the future an interesting option, given that, at the moment, there are no compelling experimental data in favour of a linearly realized spontaneous symmetry breaking mechanism. 


\section{References}

[1] R. Ferrari and A. Quadri, JHEP 0411 (2004) 019 [arXiv:hep-th/0408168].

[2] For a recent review see e.g. H. Ruegg and M. Ruiz-Altaba, Int. J. Mod. Phys. A 19 (2004) 3265 [arXiv:hep-th/0304245].

[3] D. Bettinelli, R. Ferrari and A. Quadri, "A Massive Yang-Mills Theory based on the Nonlinearly Realized Gauge Group,” arXiv:0705.2339 [hep-th], to appear in Phys. Rev. D.

[4] D. Bettinelli, R. Ferrari and A. Quadri, "One-loop Self-energy and Counterterms in a Massive Yang-Mills Theory based on the Nonlinearly Realized Gauge Group,” arXiv:0709.0644 [hep-th].

[5] R. Ferrari, JHEP 0508 (2005) 048 [arXiv:hep-th/0504023].

[6] R. Ferrari and A. Quadri, Int. J. Theor. Phys. 45 (2006) 2497 [arXiv:hep-th/0506220].

[7] R. Ferrari and A. Quadri, JHEP 0601 (2006) 003 [arXiv:hep-th/0511032].

[8] D. Bettinelli, R. Ferrari and A. Quadri, "A comment on the renormalization of the nonlinear sigma model," arXiv:hep-th/0701197, to appear in Int. J. Mod. Phys. A.

[9] D. Bettinelli, R. Ferrari and A. Quadri, JHEP 0703 (2007) 065 [arXiv:hep-th/0701212].

[10] D. Bettinelli, R. Ferrari and A. Quadri, "Gauge Dependence in the Nonlinearly Realized Massive SU(2) Gauge Theory," presented at Baltic-Nordic Workshop Network: Algebra, Geometry, and Mathematical Physics, Goteborg, Sweden, 11-13 Oct 2007, arXiv:0712.1410 [hep-th]. 